\title{
Frequency and precision of aerial surveys for kangaroo management
}

\author{
A. R. Pople \\ The Ecology Centre, School of Integrative Biology, University of Queensland, Brisbane, \\ Qld 4072, Australia. Present address: Invasive Plants and Animals, Biosecurity Queensland, \\ Department of Primary Industries and Fisheries, GPO Box 46, Brisbane, Qld 4001, Australia. \\ Email: tony.pople@dpi.qld.gov.au
}

\begin{abstract}
The appropriate frequency and precision for surveys of wildlife populations represent a trade-off between survey cost and the risk of making suboptimal management decisions because of poor survey data. The commercial harvest of kangaroos is primarily regulated through annual quotas set as proportions of absolute estimates of population size. Stochastic models were used to explore the effects of varying precision, survey frequency and harvest rate on the risk of quasiextinction for an arid-zone and a more mesic-zone kangaroo population. Quasiextinction probability increases in a sigmoidal fashion as survey frequency is reduced. The risk is greater in more arid regions and is highly sensitive to harvest rate. An appropriate management regime involves regular surveys in the major harvest areas where harvest rate can be set close to the maximum sustained yield. Outside these areas, survey frequency can be reduced in relatively mesic areas and reduced in arid regions when combined with lowered harvest rates. Relative to other factors, quasiextinction risk is only affected by survey precision (standard error/mean $\times 100$ ) when it is $>50 \%$, partly reflecting the safety of the strategy of harvesting a proportion of a population estimate.
\end{abstract}

\section{Introduction}

In wildlife management, the appropriate survey frequency and precision has generally been considered with the aim of detecting trends (Caughley 1979; Harris 1986; Gerrodette 1987). In kangaroo management, harvest regulation is primarily through quotas that are set as proportions of absolute estimates of population size (Pople and Grigg 1998). Management action is rarely taken in response to trends, so they are of secondary importance. The proportional harvesting strategy has been well studied and is considered relatively safe and efficient for a fluctuating population (Caughley 1987; Engen et al. 1997; McLeod and Pople 1998). However, the strategy requires a regular estimate of abundance on which to set a quota. Imprecision in population estimates and infrequent estimates will risk applying a quota that is either too high or too low. By not harvesting at the rate set by management, costs may be incurred to the kangaroo industry through reduced and more variable yield, to farmers through increased competition with livestock and damage to crops and there may be a social cost if kangaroos are reduced below some arbitrarily low density (i.e. quasiextinction) (Pople and Grigg 1998). These costs must be balanced against the cost of more intensive and frequent surveys (i.e. there is a trade-off) undertaken by government conservation agencies and presently largely cost-recovered from the kangaroo industry. Alternative harvest strategies can also be considered in order to reduce the risk of over- or underharvest. These include harvesting at a different, and even variable, rate, regulating effort or incorporating spatial reserves where there is no harvesting.

Kangaroo managers set annual quotas for a calendar year that represent a percentage (e.g. 15\%) of the population estimated annually (in most areas) by aerial survey in the winter of the previous year. Even if the quotas are taken, the actual rates of harvest will differ from $15 \%$ because populations will rarely remain stable. There is therefore some risk of over- or underharvest. If the population halves over 12 months, the actual harvest rate over the year becomes $\sim 21 \%$ instead of the desired (set by quota) rate of $15 \%$. If the decline is $80 \%$ (see below), the annual harvest rate is likely to be $\sim 34 \%$. A doubling of the population will result in a harvest rate of $\sim 11 \%$. These actual rates are approximate because they assume constant geometric growth and decline in the population.

In most years, this risk of over- or underharvest is likely to be small, because year-to-year fluctuations are relatively small. On a broad scale, increase in the population will be constrained physiologically by the reproductive capacity of females, and modified by sex ratio and age structure. The maximum rate of increase, even in a female-biased population with few juveniles, will fall short of doubling (Bayliss 1985; Pople 1996). For a red kangaroo population with a stable age distribution, $r_{\mathrm{m}}$ is $\sim 0.25$ (Pople 1996). However, the rate of decline can be more pronounced as it is unconstrained by an animal's physiology. Changes in population size (i.e. rate of increase) of kangaroos are closely linked to pasture biomass that is driven largely by rainfall in arid areas (e.g. Bayliss 1985; Cairns and Grigg 1993). Because there is an upper limit to a population's rate of increase, the difference between desired and actual harvest rate is potentially greater during drought than during times of plenty. Moreover, quotas are more likely to be taken during drought because animals are more accessible, graziers are more active in having animals culled and the kangaroo industry will have a relatively greater capacity to take animals as a result of previous higher population densities. In the drought of 1982-83, kangaroos declined by $\sim 40 \%$ over 12 months in the sheep rangelands 
of eastern Australia (Caughley et al. 1985). However, most of this decline occurred over a shorter period of perhaps four months (Robertson 1986), when possibly the more vulnerable individuals died. Had this period of decline been maintained, the decline over 12 months would have been $80 \%$.

Risks of over- or underharvest will be exacerbated by uncertainty in population size, which is measured by the standard error (s.e.) or confidence interval of the population estimate. For example, population size may be overestimated through chance (i.e. sampling error) alone, resulting in an effective harvest rate higher than the desired $15 \%$. This potential overharvest will be compounded if the population then declines.

The discussion above ignores the different responses a population may have to harvesting depending on whether it is increasing or decreasing. Harvest mortality can be additional to natural mortality or compensatory (Anderson and Burnham 1976; Burnham et al. 1984; Bartmann et al. 1992). For large mammals, harvest mortality will be compensated, to some extent, by lowered natural mortality rates and increased fecundity rates because these rates are density dependent (Fowler 1987). If animals that are harvested would have died anyway then the compensation could potentially be complete, but in practice compensation is only likely to be partial. To assist harvest management of ducks in the United States, the extent to which hunting mortality of ducks is compensatory is modelled and model parameters adjusted through adaptive management (Nichols et al. 1995; Williams et al. 2002). For a fluctuating population of herbivores, harvest mortality will tend to be additive during population increases when pasture is abundant, but tend to be compensatory during declines in drought (Pople 1996). Harvesting will therefore have a greater effect on a population's rate of increase when it is increasing than during drought. Harvesting is unlikely to be completely compensatory or completely additive as there will be potential survivors of drought that may be harvested and there is invariably natural mortality even when food is abundant. The relevance of compensatory mortality to this discussion is that, while the disparity between desired and actual harvest rates is greatest during declines, the overall impact of harvesting is less during declines.

Using a risk-assessment framework, this paper attempts to identify the appropriate survey frequency and precision for kangaroo harvest management in two locations spanning a broad range of annual rainfall. This range in aridity roughly covers that experienced across the area where kangaroos are commercially harvested in Australia (Pople and Grigg 1998).

\section{Methods}

There is uncertainty from several sources in the future dynamics of kangaroo populations. There is uncertainty in the environment (process uncertainty), in the response of kangaroos to the environment and harvesting (model uncertainty) and also in the density of kangaroos estimated from aerial survey (observation uncertainty). Therefore, simulation modelling was used to assess risk (Burgman et al. 1993) in harvest management of kangaroo populations and included all three forms of uncertainty. Scenarios covering the following factors were examined:

(1) two alternative population models (model uncertainty),

(2) an arid and a more mesic environment (process uncertainty),
(3) harvest rates ranging from 0 to $20 \%$ (no uncertainty in harvest size),

(4) survey frequency varying from 1 to 5 years (observation uncertainty), and

(5) precision (s.e./mean $\times 100$ : Andrew and Mapstone 1987) of population estimates varying over $0-200 \%$ (observation uncertainty)

The additional risk posed to the population through harvesting is couched here primarily in terms of probability of quasiextinction (i.e. population falling below an unacceptably low threshold density). The effect of survey frequency and precision on variation in population size and harvest offtake was also considered here. However, other related response variables could have been used, including minimum and average population density, time spent below particular threshold densities, average harvest offtake, or average pasture biomass.

Quasiextinction is not just a theoretical concept nor is the threshold density necessarily arbitrary. It is likely to be of principal concern to the kangaroo industry, as well as landholders and conservationists, because harvesting is unlikely to be commercially viable below some threshold density. Hacker et al. (2003) suggested that this is $\sim 5$ kangaroos $\mathrm{km}^{-2}$. However, in practice there are regions where harvesting occurs at densities as low as 2-3 kangaroos $\mathrm{km}^{-2}$, such as in the north-west of the South Australian Pastoral Zone (Cairns and Grigg 1993).

Two models describing the dynamics of kangaroo populations in arid areas were considered: an interactive model described by Caughley (1987) and a ratio-dependent model developed by McCarthy (1996). In both models, kangaroo density has a negative effect on rate of increase and so harvest mortality is partially compensated. Conversely, rainfall positively influences rate of increase in both models so that harvest mortality tends to be additive during increases and compensatory during declines. Other models could have been used, in particular a Ricker-type model (e.g. Jonzen et al. 2005), but McCarthy (1996) recorded lower risks of overharvesting with this model compared with the ratio-dependent model, so it was not considered.

In the interactive model, rainfall drives pasture biomass, which in turn determines the rate of increase of the kangaroo population. There are two negative feedback loops. The first is pasture biomass reducing pasture growth. The second is kangaroo density reducing kangaroo rate of increase by reducing the available biomass by eating it. The interactive model can be described by the following equations:

$$
\begin{gathered}
r=-1.6+2\left(1-\mathrm{e}^{-0.007 V}\right) \\
\Delta V=-55.12-0.01535 V-0.00056 V^{2}+2.5 R T \\
I=86\left(1-\mathrm{e}^{-V / 34}\right) \times W^{0.75} \times(1+S)
\end{gathered}
$$

where $r$ is the annual exponential rate of increase of kangaroos, $V$ is pasture biomass $\left(\mathrm{kg} \mathrm{ha}^{-1}\right)$ at the start of the interval, $\Delta V$ is the growth increment in pasture biomass over three months and $R T$ is rainfall $(\mathrm{mm})$ over three months. $I$ is intake $(\mathrm{g})$ of pasture by kangaroos and sheep per day, $S$ is the amount of pasture eaten by sheep relative to kangaroos and $W$ is the average bodyweight ( $\mathrm{kg}$ ) of a kangaroo, assumed here to be $26 \mathrm{~kg}$, which is more typical of a harvested kangaroo population than the $35 \mathrm{~kg}$ assumed by Caughley (1987) (Grigg 2002). Taking Caughley's 
(1987) approach, the kangaroo population was harvested and pasture grew and was grazed down in weekly time steps. Pasture growth was drawn from a normal distribution with a standard deviation of $52 \mathrm{~kg} \mathrm{ha}^{-1}$, as estimated by Robertson (1987). Sheep competed with the kangaroo population, consuming 1.5 times the pasture eaten by kangaroos (i.e. $S=1.5$ ), as suggested by Caughley (1987). Including sheep added realism by reducing the average density of the kangaroo population and associated harvest offtake, but did not alter its dynamics, so an accurate estimate of $S$ is not critical.

The ratio-dependent model is much simpler, with the rate of increase of kangaroos a function of the ratio of rainfall to population size. McCarthy's (1996) original parameterisation used a 10-year time series for the whole pastoral zone in South Australia and ignored harvest offtake. The model was refitted, using non-linear least-squares regression, to a much longer time series from a subset of that area, comprising 25 years (1978-2002) of rainfall and red kangaroo harvest offtake and density from north-east South Australia. The resulting model can be described by the following equation:

$$
r=a-b \times \mathrm{e}^{(-c \times R A / D)}-H R
$$

where $r$ is the annual exponential rate of increase of kangaroos, $R A$ is the rain $(\mathrm{mm})$ that fell in the 12 months before the start of the period covered by $r, D$ is population density at the start of that period, $H R$ is the instantaneous harvest rate over the period (see Jonzen et al. 2005) and $a, b$ and $c$ are parameters to be estimated. For simulations with the ratio-dependent model, the kangaroo population was harvested in three-monthly time steps.

In both models, harvesting was introduced before reproduction and natural mortality, which are modelled together as $r$. This can be described as:

$$
D_{t+1}=\left(D_{\mathrm{t}}-H\right) \times \mathrm{e}^{r}
$$

where $r$ is the modelled exponential rate of increase between time $t$ and $t+1, D_{\mathrm{t}}$ and $D_{t+1}$ are population densities at time $t$ and $t+1$, respectively, and $H$ is harvest offtake at the start of the period.

Both models are based on data for red kangaroos (Macropus rufus), so extrapolation of the results to the other harvested species, eastern grey kangaroos ( $M$. giganteus), western grey kangaroos (M. fuliginosus) and common wallaroos (M. robustus), needs to be made cautiously. In particular, the two grey kangaroo species have lower maximum rates of increase than red kangaroos and perhaps higher rates of decline in drought in arid regions (Robertson 1986; Bayliss 1987). However, the qualitative differences between the species' dynamics are slight, suggesting that the qualitative results from the modelling should apply to all four species in an arid environment. Management agencies accommodate species' differences in lower quotas for grey kangaroos as a percentage of the population.

Contrasting the results of the two models addresses just one form of model uncertainty. A range of parameters for each model can also be considered. This was not done here for two reasons. First, overall model structure was considered a greater influence. Second, for the interactive model, the maximum rate of decline was parameterised from a four-month period observed in the drought of 1982-83 (Caughley 1987). Whether this rate of decline could be maintained over a longer period is presently unknown, but a model allowing this should provide conservative estimates of risk (i.e. tend to overestimate risk).

Both the interactive and ratio-dependent models require rainfall as the main input variable, which was introduced into each model seasonally (i.e. three monthly). Rainfall is non-negative and its distribution is right skewed and so was drawn from a lognormal distribution. An arid-zone environment was represented by rainfall parameters from Menindee Post Office in western New South Wales (annual mean $=244 \mathrm{~mm}$, standard deviation $($ s.d. $)=106 \mathrm{~mm}$ ), where Caughley's (1987) interactive model was parameterised. For the ratio-dependent model, Menindee rainfall needed to be rescaled to north-east South Australia $($ mean $=195 \mathrm{~mm}$, s.d. $=95 \mathrm{~mm})$, by converting it to standard deviation units then multiplying by 95 and adding 195 . To make the results of both models comparable, average kangaroo density needed to be similar in both models when unharvested. Density in the ratio-dependent model was therefore rescaled by multiplying it by the average unharvested density of the interactive model divided by the average unharvested density of the ratio-dependent model.

Although the interactive model was developed for an aridzone population of red kangaroos, it can yield results relevant to eastern grey kangaroos in a more mesic environment. First, predictions of such entities as harvest rates for maximised yield, average population size and harvest yield were not sought; rather, the objective of the modelling was to compare management scenarios. It is the qualitative rather than the quantitative results of the modelling that are of relevance. Nevertheless, the modelled population needed to show similar dynamics and a similar response to harvesting to an eastern grey kangaroo population in the east of the sheep rangelands. The population fluctuations in Caughley's (1987) interactive model therefore required dampening by reducing the standard deviation in seasonal rainfall by $37 \%$ so that it was equivalent to that in the more mesic environment around Yass (annual mean rainfall $=652 \mathrm{~mm}$, s.d. $=178 \mathrm{~mm}$ ) in south-eastern New South Wales where eastern grey but not red kangaroos occur. Altering the standard deviation in rainfall and not the mean avoided the 'paradox of enrichment', which would have not just increased the mean kangaroo density but also the amplitude of fluctuations in kangaroo density (Crawley 1983).

In both models, instantaneous harvest rates were converted to isolated rates of harvesting (Caughley 1977), appropriate to the time step, to simulate harvesting spread evenly throughout each year. The models predicted population size at least every three months, mimicking true population size with no observation error, and estimated population size annually, mimicking a survey with observation error. Harvest offtake for each three months was determined as a proportion of the most recent population estimate. Harvest offtake was therefore the same for each three months of the year, despite population size changing at each three-monthly time step. Population estimates were drawn from a log-normal distribution, appropriate for non-negative densities (e.g. Holmes 2001; Dennis et al. 2006), with a standard deviation equal to the survey precision multiplied by the predicted population density. Survey precision varied from 0 to $200 \%$, but was set at $20 \%$ unless otherwise stated.

The initial population size for both models was 10 kangaroos $\mathrm{km}^{-2}$ and initial pasture biomass for the interactive model was 
$300 \mathrm{~kg} \mathrm{ha}^{-1}$, representing roughly the average density and pasture biomass over 100-year simulations of the interactive model with a $10 \%$ harvest. Annual yield curves and associated densities were first constructed for both the interactive and ratio-dependent models with no observation error (i.e. precision $=0$ ) using 1000 simulations of 100-year periods. Yield curves identify the long-term, annual harvest offtake that will be obtained from a given harvest rate. To assess quasiextinction risk, each simulation was run for 20 years, representing a likely period of concern for managers and encompassing the period of 10 years or so that past kangaroo density will influence subsequent density (Caughley 1987). For each scenario (i.e. combinations of factors in 1-5 above), the average of 1000 simulations is reported.

These simulations assume that quotas remain constant between surveys. There are several examples from kangaroo management where quotas have been adjusted between infrequent (i.e. $>1$-year interval) surveys. In the northern tablelands of New South Wales, quotas have been adjusted for changes in kangaroo numbers in adjoining management zones and for recent rainfall (Gilroy 1999). Quotas have been similarly adjusted in Western Australia where the harvest area was surveyed triennially, but now there are three survey areas with a different area surveyed each year (Pople and Grigg 1998). The risks of overharvest described by these simulations are therefore worst-case scenarios. A simple rule was incorporated into the simulations whereby a survey was undertaken at a set frequency of three or five years unless rainfall in the previous year had been $\geq 50 \%$ below average, in which case the population was surveyed.

Population models were run in Excel with the add-in POPTOOLS (Greg Hood, CSIRO 2002, http:/www.dwe.csiro.au/ vbc/poptools/index.htm).

\section{Results}

\section{Differences between population models}

The ratio-dependent model was a good fit to the data $\left(R^{2}=0.63\right)$, similar to the fit reported by McCarthy (1996). The ratio-dependent model of best fit had parameters $( \pm$ s.e. $)$ of $a=0.538( \pm 0.197), b=1.546( \pm 0.437)$ and $c=0.079( \pm 0.046)$. Density in the ratio-dependent model needed to be multiplied by 1.154 to make the average unharvested densities of the two models comparable.

The two models of population dynamics display quite different population behaviour despite both fitting data from the South Australian Pastoral Zone reasonably well (McCarthy 1996; Pople 2006). The interactive model generates greater amplitude in population size over time than the ratio-dependent model, resulting from weaker density dependence. In the ratiodependent model, the population recovers relatively rapidly from declines in drought and population eruptions are similarly tempered.

Furthermore, the ratio-dependent model predicted smaller declines during drought. This disparity is the result of different rainfall periods ultimately driving population rate of change of kangaroos in the two models. For the interactive model, kangaroos essentially respond to rainfall without any lag. In the ratiodependent model, there is a 12-month lag in the response.
A 12-month lag may be appropriate where enhanced juvenile survival is not detected immediately by aerial survey, but it is implausible during drought where there is substantial adult mortality that would be (and has been) detected immediately by aerial survey. A final difference between the two models is that the ratio-dependent model generates little effect of harvesting over the range of $0-20 \%$ on population decline, again resulting from its stronger density dependence. The differences between the models are clearly seen in the yield curves (Fig. 1), with the ratiodependent model indicating much higher sustainable harvests than the interactive model and a highly implausible maximum sustained yield (MSY) of $>30 \%$, representing the harvest rate associated with the maximum point of the yield curve.

Overall, these differences result in a lower risk of overharvest in the ratio-dependent model, particularly during drought. Therefore, the risk assessment was undertaken solely with the more realistic and conservative interactive model.

\section{Contrasting environments}

Not surprisingly, average yield at Yass was higher than at Menindee and the maximum sustained yield is pushed slightly to the right ( $\sim 13 \%$ for Menindee and $\sim 15 \%$ for Yass) (Fig. $2 a$ ). These yield curves and the associated population dynamics appear plausible for both environments, although the fluctuations in kangaroo numbers in more mesic environments have been poorly described in the literature. Modelled average density over 100 years is higher at Yass and declines non-linearly with harvest rate (Fig. $2 b$ ). It obviously follows that the probability of the population falling below some low density increases with increasing harvest rate. Though not shown, risk does not increase linearly with harvest rate, with risk accelerating from 10 to $15 \%$ and decelerating from 15 to $20 \%$, which coincides with the MSY for these models of 10-15\% (Fig. 2a).

\section{Survey frequency and harvest strategy}

As expected, the risk of quasiextinction is lower at Yass (Fig. 3a) than at Menindee (Fig. $3 b$ ). Reducing survey frequency not surprisingly increases the probability of quasiextinction. Although the increase does not appear dramatic, a five-year survey frequency at Yass returns a risk of $\sim 0.1$ of the population falling

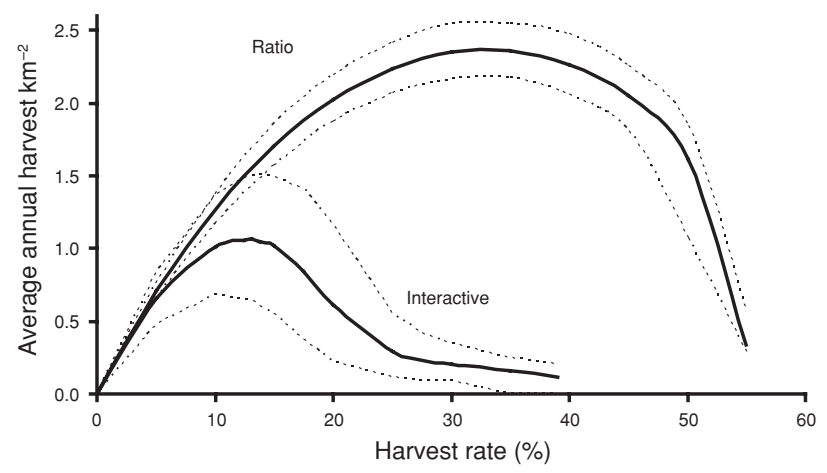

Fig. 1. Yield curves based on the ratio-dependent model and the interactive model of red kangaroo population dynamics where the population is harvested annually at constant rates ranging from 0 to $55 \%$ over 100 years. Solid lines represent the average of 1000 simulations and $95 \%$ confidence intervals are shown as dotted lines. 
below 2 kangaroos $\mathrm{km}^{-2}$ and $\sim 0.2$ for a threshold of 5 kangaroos $\mathrm{km}^{-2}$. The equivalent risks for an annual survey frequency are $<0.01$ and 0.1 , respectively.

An alternative to the probability of quasiextinction shown in Fig. 3 is the time (e.g. number of months) spent below particular thresholds. The pattern is similar, but allows an acceptable threshold to be identified in different terms. For example, it may not be cost-effective for harvesters to operate at densities below $\sim 3$ kangaroos $\mathrm{km}^{-2}$. The risk curve can then be used to identify the likely period when harvesting will not be possible. Alternatively, harvesters may simply want to avoid densities below a particular threshold and there may be some sociopolitical cost of dropping below some density, in which case Fig. 3 is appropriate.

If the harvest rate is reduced to $10 \%$, then the risk of quasiextinction is greatly reduced (Figs $4 a, b$ ). It is only at the lower threshold of $\sim 3$ kangaroos $\mathrm{km}^{-2}$ that reducing survey frequency with a $10 \%$ harvest becomes riskier than a $15 \%$ harvest with annual surveys. Even then, it is only when surveys are as infrequent as every five years. On average, reducing the harvest rate from $15 \%$ to $10 \%$ results in an increase in average population density of $30-50 \%$ and a reduction in average harvest offtake of $1-13 \%$ (Fig. $2 a, b$ ).
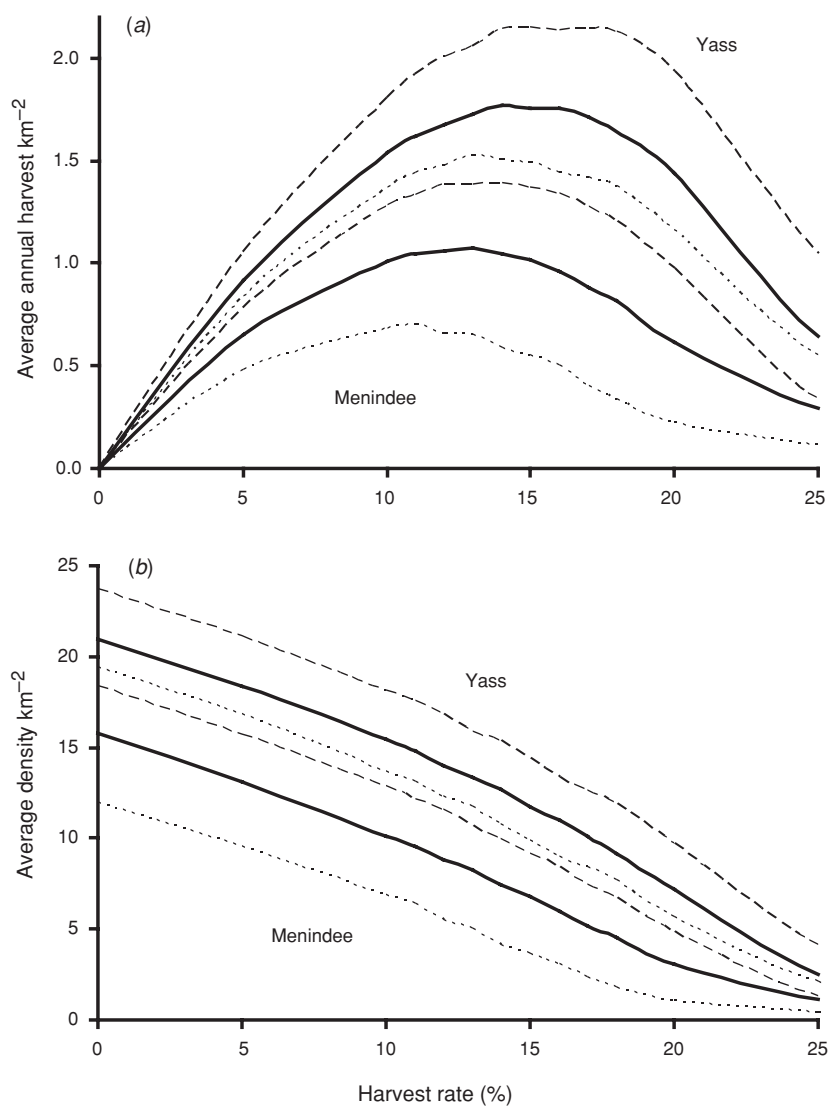

Fig. 2. (a) Yield curves and (b) average kangaroo densities for Menindee and Yass based on simulations over 100 years of a kangaroo population harvested annually at constant rates ranging from 0 to $40 \%$. Solid lines represent the average of 1000 simulations and $95 \%$ confidence intervals are shown as dotted (Menindee) or dashed (Yass) lines.
Reducing survey frequency results in only a slight increase in population and harvest variability and has little effect on average harvest offtake and population density. At Menindee, harvest coefficient of variation (i.e. s.d./mean $\times 100$ ) increases in a sigmoidal fashion from $41 \%$ to $55 \%$ as survey frequency is increased from one to five years.

Always conducting surveys when rainfall was $\geq 50 \%$ below average made little difference to quasiextinction risk compared with surveys every three or five years at Yass, but there was some reduction in risk for the 5-year survey program at Menindee (Fig. $5 a, b$ ). This presumably reflects the greater likelihood of rainfall $\geq 50 \%$ below average at Menindee.

\section{Survey precision}

Pople (2003) found that precision of $\leq 50 \%$ had little effect on the probability of quasiextinction underharvesting. Here, a broader range of possible precisions and drawing population size from a log-normal rather than a normal distribution identifies when there is a substantial risk (Fig. $6 a, b$ ). Above 50\%, precision increases the probability of quasiextinction dramatically. Interestingly, there is an interaction between survey precision and frequency (Fig. 7a, $b$ ). Reducing survey frequency from every one to every five years when precision is $50 \%$ has a much greater impact on quasiextinction risk than if precision is only $20 \%$.

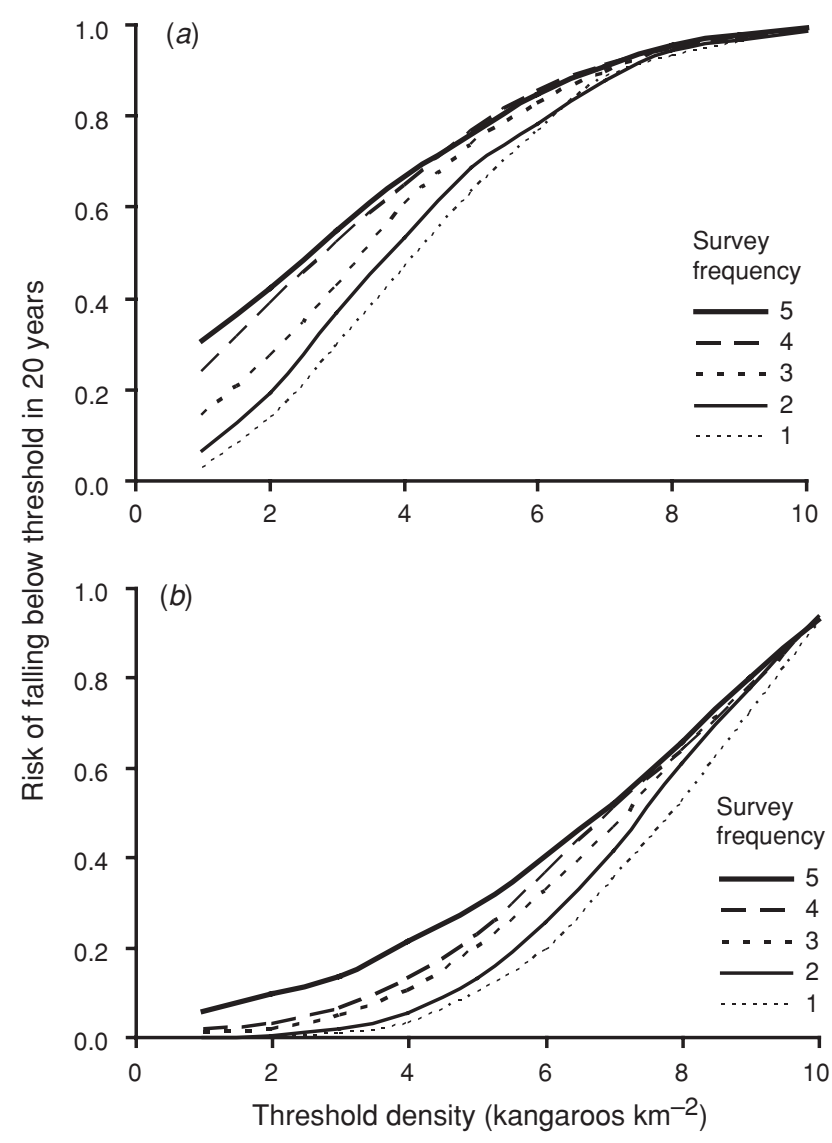

Fig. 3. Probability of quasiextinction over 20 years from a harvest rate of $15 \%$ with a survey frequency of $1-5$ years for $(a)$ Yass and $(b)$ Menindee. 
With poorer precision, population size becomes more variable, as the actual harvest rate is less likely to be the desired $15 \%$ (Fig. 8). Harvest variation increases much more markedly with worsening precision (Fig. 8). Interestingly, the lines for the two locations converge at extremely poor precision (i.e. high s.e./mean), as the observation error becomes an increasingly greater influence on harvest and population variation than process variation, driven primarily by rainfall.

\section{Trade-off between survey frequency and cost}

The appropriate survey frequency will depend on the costs of the surveys and the costs of compromising the harvest strategy by not always knowing population size. Imprecision can also incur costs, but this seems a problem in this case only when precision is $>50 \%$ (Figs 6,7 ). To make costs comparable, the potential gains and losses incurred by the kangaroo industry, graziers, conservationists and any other stakeholders from adopting various monitoring programs need to be put onto the same scale. This would require detailed exploration and so is not determined here. Instead, a simple estimate of survey cost is contrasted with quasiextinction risk for increasing survey frequency. This should provide a first approxima-

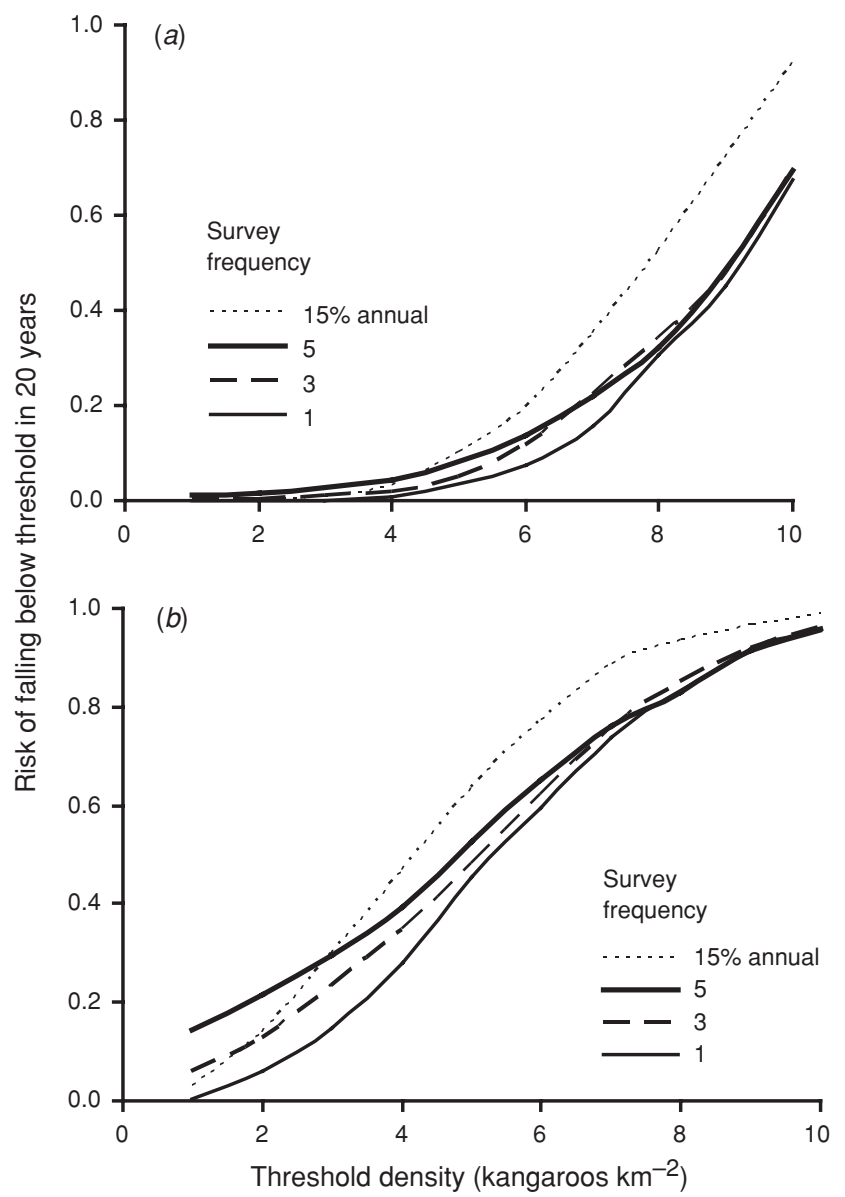

Fig. 4. Probability of quasiextinction over 20 years from a harvest rate of $10 \%$ with a survey frequency of 1-5 years for $(a)$ Yass and $(b)$ Menindee. The risk for a $15 \%$ harvest with an annual survey frequency (Fig. 3) is shown as a dashed line. tion of the appropriate survey frequency and the nature of the trade-off.

There are fixed costs associated with conducting aerial surveys that do not change if surveys are conducted less frequently. These include labour costs and can be assumed to be $\$ 50000$ per annum. The variable costs primarily comprise aircraft running costs and are assumed to be $\$ 50000$ per survey. The total five-year survey cost (fixed + variable $\times$ no. of surveys) is shown in Fig. 9. This shows the ever-diminishing savings from reducing survey frequency. Quasiextinction risk from Figs 3 and 4 is also shown in Fig. 9, but only for a threshold density of 2 kangaroos $\mathrm{km}^{-2}$. Risk of quasiextinction increases markedly beyond a survey frequency of every 3-4 years. The actual probability should be interpreted

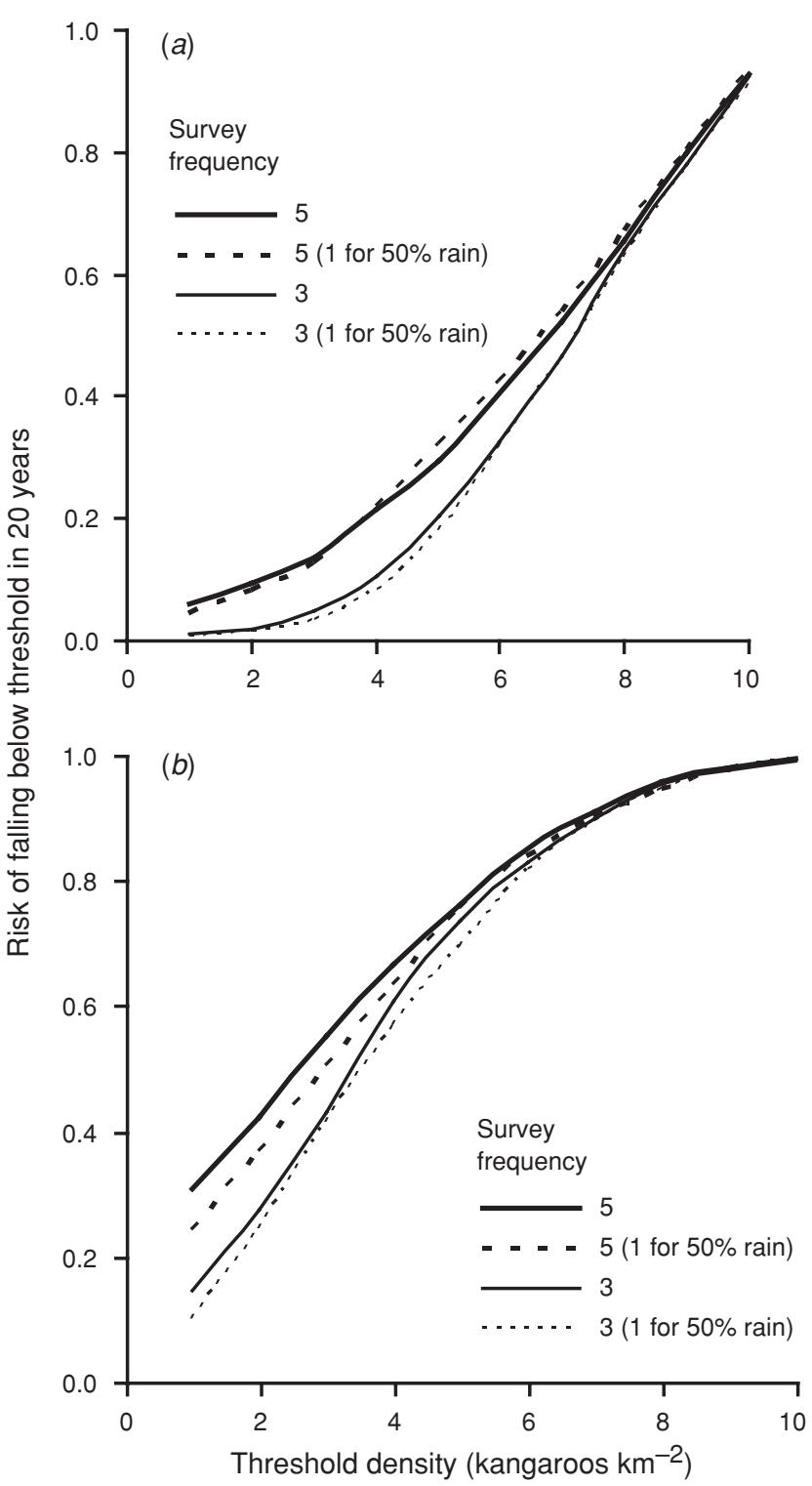

Fig. 5. Probability of quasiextinction over 20 years from a harvest rate of $15 \%$ with a survey frequency of three or five years (solid lines) and annual if rainfall is $<50 \%$ of the long-term mean (dotted lines) for (a) Yass and (b) Menindee. 
cautiously as it is dependent on the model structure and parameters and these have not been assessed for this population. Furthermore, the acceptable level of risk is a value judgement.

\section{Discussion}

There are several management recommendations that are reasonably clear from this risk assessment. First, in more mesic environments, a survey frequency of three years with a precision of $20 \%$ appears to increase the risk of overharvest only slightly over annual surveys, while substantially reducing survey costs (Fig. 9). Whether the increase in risk is acceptable is a decision for management in consultation with stakeholders. Second, in arid environments, harvesting near the MSY (e.g. 15\% for the interactive model) ideally requires annual estimates of population size with a precision of $<50 \%$ (Figs 6,7 ). In some regions, such as north-west South Australia and north-west Queensland, harvest rates are low (Pople 2006), questioning the need for annual surveys. Fig. 4 suggests that reducing the quota to $10 \%$ would largely offset the quasiextinction risks resulting from surveying less frequently. Furthermore, under this regime of survey frequency and precision, variability in population size and harvest offtake will not differ greatly from a regime of annual surveys and population density estimated with certainty (Fig. 8).

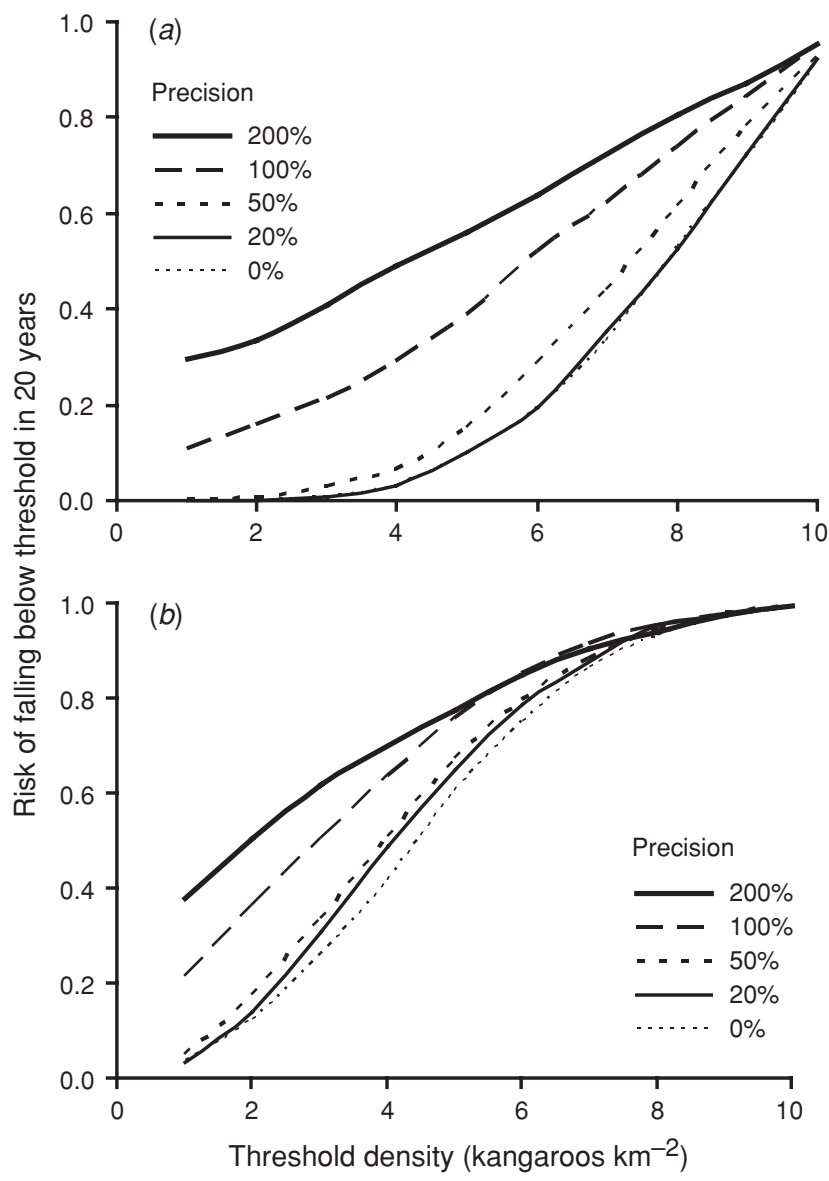

Fig. 6. Probability of quasiextinction over 20 years from a harvest rate of $15 \%$ with a precision (s.e./mean $\times 100$ ) of $0-200 \%$ for $(a)$ Yass and (b) Menindee.
The MSYs for these models do not necessarily translate to appropriate harvest rates to set as quotas. The harvest is strongly male-biased, which shifts the MSY to a higher rate (Hacker et al. 2003). The relevant point from the modelling is that a reduction in harvest rate has a greater influence on risk than changing survey frequency (Figs 4, 9), at least given the model structure and range of parameters examined here.

Increased survey frequency during drought did little to alter the quasiextinction risk in the present study (Fig. 5). This is an indication of the relative safety of the strategy of harvesting a proportion of the population that is near or below the MSY. While harvesting at a higher than desirable rate will exacerbate a population decline, the overwhelming influence will be the rainfall deficit. However, droughts are likely to have been infrequent in the 20-year simulation period and so the analysis here only provides guidance for management in the longer term, not the immediate concerns of a particular drought. Using simulation, Pople (2003) found that 6-monthly rather than annual surveys during a major drought made little difference to the percentage decline in population size over the initial 12 months, but nevertheless increased the mean minimum density to which simulated populations declined over

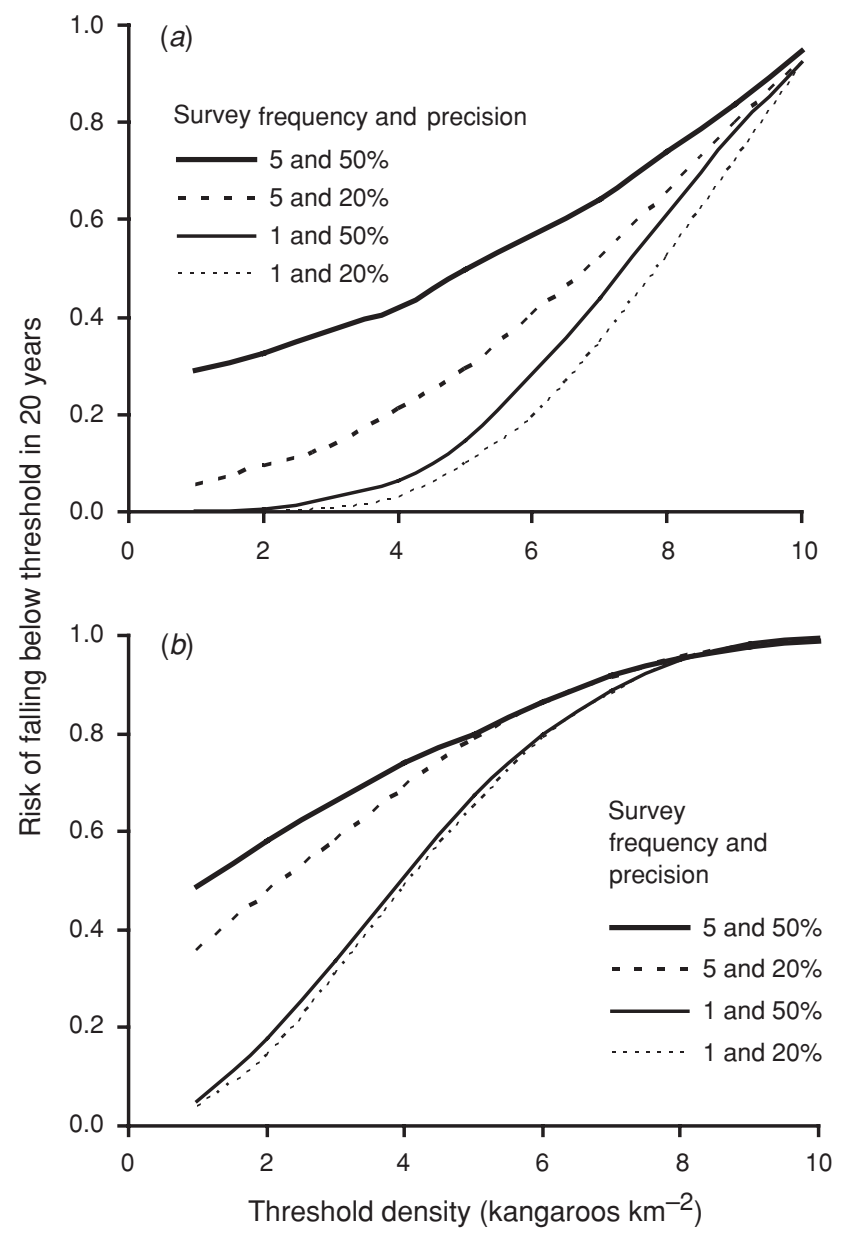

Fig. 7. Probability of quasiextinction over 20 years from a harvest rate of $15 \%$ with a precision (s.e./mean $\times 100$ ) of $20 \%$ or $50 \%$ and a survey frequency of one or five years for $(a)$ Yass and $(b)$ Menindee. 


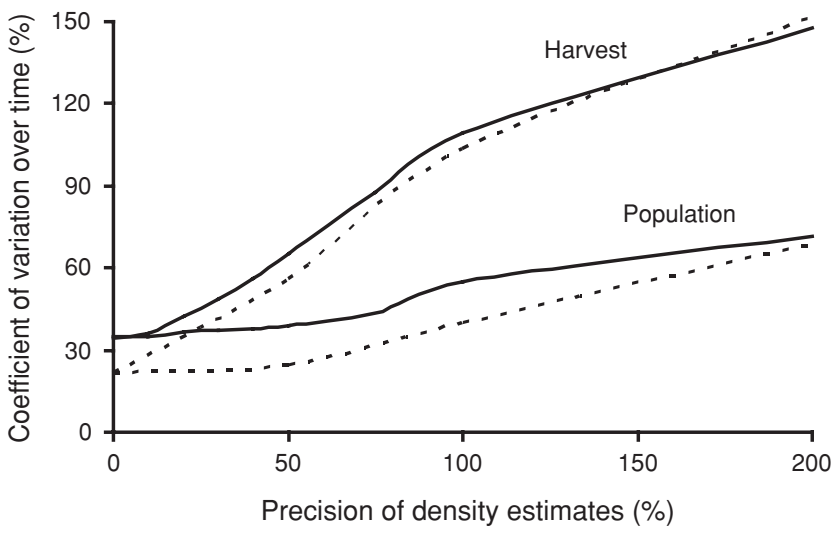

Fig. 8. Coefficient of variation $($ s.d./mean $\times 100)$ of population density and harvest offtake over 20 years for Yass (dashed line) and Menindee (solid line) for precision (s.e./mean $\times 100$ ) varying between 0 and $200 \%$, a harvest rate of $15 \%$ and an annual survey frequency.

the subsequent decade. However, the same effect could have been achieved by reducing the harvest rate from $15 \%$ to $8-10 \%$, consistent with the more general result reported here. The question is whether the decline could be predicted with confidence. Rainfall is a useful predictor of likely population decline in drought, but a poor predictor outside of drought (Pople 2006). This latter observation is because of variation in pasture response to rainfall and because food may not be the primary limiting factor outside of drought. The ability of rainfall to predict rate of increase in kangaroos can be gauged by the convex shape of the numerical response of kangaroos, involving steep declines in drought but relatively little change in the rate of increase of kangaroos when rainfall is above average (Davis et al. 2002).

The insensitivity of risk to changes in precision of less than $50 \%$ is surprising (Fig. 6). It again partly reflects the safety of the harvest strategy. Milner-Gulland et al. (2001) similarly found that their harvest strategies were unaffected by precision worsening from $20 \%$ to $40 \%$. The strategy most affected involved harvesting all individuals above a threshold density,

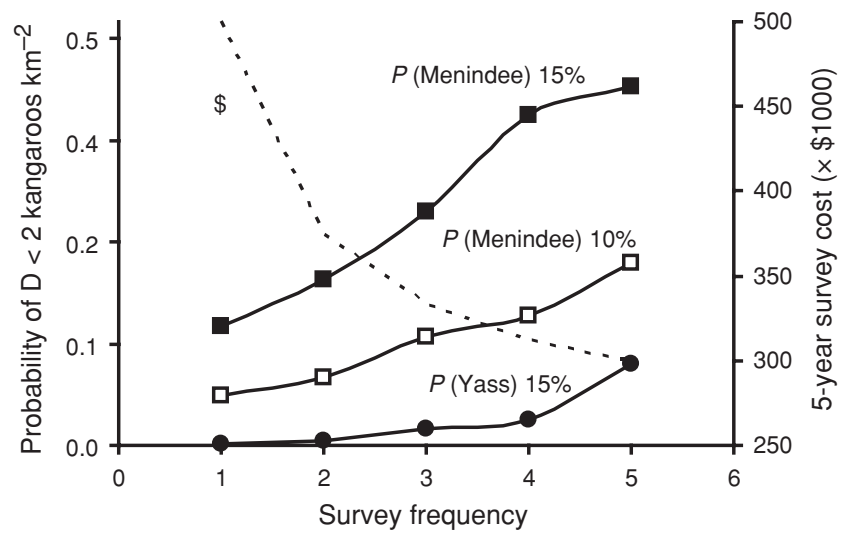

Fig. 9. Probability of quasiextinction (solid line) and survey cost over five years (dotted line) for survey frequencies of $1-5$ years for Yass (solid circles) and Menindee (solid squares: $15 \%$ harvest; open squares: $10 \%$ harvest). which was not considered here and is highly unlikely to be feasible for kangaroos on a broad scale.

An alternative approach to determining the appropriate survey frequency is to couch the problem as a decision that needs to be made each year as to whether or not to survey. Hauser et al. (2006) used such a framework to identify that, for managing an arid-zone kangaroo harvest, it was preferable to vary the survey regime on the basis of the state of the system. Kangaroo population dynamics were simulated using a stochastic, Ricker-type model that has stronger density dependence than the interactive model used here. In further contrast to the present study, neither harvest rate nor survey precision was varied. If a survey was not conducted, harvest quotas were determined from a population estimate predicted from past rainfall and population size, in contrast to the constant population size assumed in this study. The optimal decision was found by maximising 'utility', which was a non-linear function (roughly an inverted parabola) of density. This was traded-off against the cost of a survey, which was in the same currency. The function described a desirable (i.e. positive utility) density range, with less desirable densities above and very undesirable densities below that range. Thus, both quasiextinction and damage from overgrazing at high densities were considered as costs. Not surprisingly, as the predicted population size became less certain, because of above-average rainfall or increasing time since the last survey, a survey was more valuable. These results are consistent with the findings of the present study, where the increased uncertainty associated with a more arid environment required an increased survey frequency to keep quasiextinction risk constant. Nevertheless, Hauser et al. (2006) found that a flexible monitoring regime was preferred over monitoring at fixed intervals. In addition to uncertainty in previous population estimates, estimates near the upper and lower threshold for desirable density increased the need for a survey. This result will be influenced by the ability to predict density with a model and to have correctly identified the desirable density range.

In addition to lower harvest rates, other harvest strategies could be employed to offset the risks associated with reduced survey frequency. It is well recognised that imposing a threshold density below which there is no harvest will reduce the probability of quasiextinction and optimise harvest offtake if coupled with increasing harvest rates above the threshold (Engen et al. 1997; Milner-Gulland et al. 2001). The problem is that the temporal variation in harvest offtake increases with the density at which the threshold is set, and this increase is exponential for kangaroos, as modelled by Pople (2003). Although a low threshold is effectively in force presently because of economic constraints, a threshold set and policed by managers would guard against an increase in the value of kangaroo products, allowing shooters to operate at lower densities.

\section{Acknowledgements}

This project was supported by an ARC SPIRT grant to Hugh Possingham, Gordon Grigg and Stuart Phinn at the University of Queensland, with financial contributions from Queensland, New South Wales, South Australian and Commonwealth conservation agencies and Packer Tanning. The manuscript was improved by comments from two anonymous referees. 


\section{References}

Anderson, D. R., and Burnham, K. P. (1976). Population ecology of the mallard. VI. The effect of exploitation on survival. U.S. Fish and Wildlife Service Resource Publication No. 128.

Andrew, N. L., and Mapstone, B. D. (1987). Sampling and the description of spatial pattern in marine ecology. Oceanography and Marine Biology: An Annual Review 25, 39-90.

Bartmann, R. M., White, G. C., and Carpenter, L. H. (1992). Compensatory mortality in a Colorado mule deer population. Wildlife Monographs 121

Bayliss, P. (1985). The population dynamics of red and western grey kangaroos in arid New South Wales, Australia. II. The numerical response function. Journal of Animal Ecology 54, 127-135. doi:10.2307/4625

Bayliss, P. (1987). Kangaroo dynamics. In 'Kangaroos: their Ecology and Management in the Sheep Rangelands of Australia'. (Eds G. Caughley, N. Shepherd and J. Short.) pp. 119-134. (Cambridge University Press: Cambridge.)

Burgman, M. A., Ferson, S., and Akcakaya, H. R. (1993). 'Risk Assessment in Conservation Biology.' (Chapman and Hall: London.)

Burnham, K. P., White, G. C., and Anderson, D. R. (1984). Estimating the effect of hunting on annual survival rates of adult mallards. Journal of Wildlife Management 48, 350-361. doi:10.2307/3801166

Cairns, S. C., and Grigg, G. C. (1993). Population dynamics of red kangaroos (Macropus rufus) in relation to rainfall in the South Australian pastoral zone. Journal of Applied Ecology 30, 444-458. doi:10.2307/ 2404185

Caughley, G. (1977). 'Analysis of Vertebrate Populations.' (Wiley and Sons: London.)

Caughley, G. J. (1979). Design for aerial censuses. In 'Aerial Survey of Fauna Populations'. pp. 15-23. (Australian Government Publishing Service: Canberra.)

Caughley, G. (1987). Ecological relationships. In 'Kangaroos: their Ecology and Management in the Sheep Rangelands of Australia'. (Eds G. Caughley, N. Shepherd and J. Short.) pp. 159-187. (Cambridge University Press: Cambridge.)

Caughley, G., Grigg, G. C., and Smith, L. (1985). The effect of drought on kangaroo populations. Journal of Wildlife Management 49, 679-685. doi: $10.2307 / 3801694$

Crawley, M. J. (1983). 'Herbivory.' (Blackwell Scientific Publications: London.)

Davis, S. A., Pech, R. P., and Catchpole, E. A. (2002). Populations in variable environments: the effect of variability in a species' primary resource. Philosophical Transactions of the Royal Society of London series B 357, 1249-1257. doi:10.1098/rstb.2002.1125

Dennis, B., Ponciano, J. M., Lele, S. R., Taper, M. L., and Staples, D. F. (2006). Estimating density dependence, process noise, and observation error. Ecological Monographs 76, 323-341. doi:10.1890/00129615(2006)76[323:EDDPNA]2.0.CO;2

Engen, S., Lande, R., and Saether, B.-E. (1997). Harvesting strategies for fluctuating populations based on uncertain population estimates. Journal of Theoretical Biology 186, 201-212. doi:10.1006/jtbi. 1996.0356

Fowler, C. W. (1987). review of density dependence in populations of large mammals. In 'Current Mammalogy'. (Ed. H. H. Genoways.) pp. 401-441. (Plenum Press: New York.)

Gerrodette, T. (1987). A power analysis for detecting trends. Ecology 68 1364-1372. doi:10.2307/1939220

Gilroy, J. (1999). Kangaroo monitoring in relation to the kangaroo management plan in New South Wales. Australian Zoologist 31, 306-308.

Grigg, G. (2002). Conservation benefit from harvesting kangaroos: status report at the start of a new millenium. In 'A Zoological Revolution.
Using Native Fauna to Assist in its Own Survival'. (Eds D. Lunney and C. Dickman.) pp. 53-76. (Royal Zoological Society of New South Wales \& Australian Museum: Sydney.)

Hacker, R. B., McLeod, S. R., and Druhan, J. (2003). Final Report for Project \#D8003. Evaluating Alternative Management Strategies for Kangaroos in the Murray Darling Basin. NSW Department of Agriculture, Dubbo.

Harris, R. B. (1986). Reliability of trend lines obtained from variable counts. Journal of Wildlife Management 50, 165-171. doi:10.2307/3801508

Hauser, C. E., Pople, A. R., and Possingham, H. P. (2006). Should managed populations be monitored every year? Ecological Applications 16, 807-819. doi:10.1890/1051-0761(2006)016[0807:SMPBME]2.0.CO;2

Holmes, E. E. (2001). Estimating risks in declining populations with poor data. Proceedings of the National Academy of Sciences of the United States of America 98, 5072-5077. doi:10.1073/pnas.081055898

Jonzen, N., Pople, A. R., Grigg, G. C., and Possingham, H. P. (2005). Of sheep and rain: large-scale population dynamics of the red kangaroo. Journal of Animal Ecology 74, 22-30. doi:10.1111/j.1365-2656. 2005.00915.x

McCarthy, M. A. (1996). Red kangaroo (Macropus rufus) dynamics: effects of rainfall, density dependence, harvesting and environmental stochasticity. Journal of Applied Ecology 33, 45-53. doi:10.2307/2405014

McLeod, S., and Pople, T. (1998). Optimal exploitation strategies for kangaroo populations in variable environments. In 'Proceedings of the 11th Australian Veterbrate Pest Conference, 3-8 May 1998, Bunbury, Western Australia'. pp. 375-380.

Milner-Gulland, E. J., Shea, K., Possingham, H., Coulson, T., and Wilcox, C. (2001). Competing harvesting strategies in a simulated population under uncertainty. Animal Conservation 4, 157-167. doi:10.1017/ S1367943001001184

Nichols, J. D., Johnson, F. A., and Williams, B. K. (1995). Managing North American waterfowl in the face of uncertainty. Annual Review of Ecology and Systematics 26, 177-199. doi:10.1146/annurev.es. 26.110195.001141

Pople, A. R. (1996). Effects of harvesting upon the demography of red kangaroos in western Queensland. Ph.D. Thesis, The University of Queensland, Brisbane.

Pople, A. (2003). Harvest management of kangaroos during drought. Unpublished report to New South Wales National Parks and Wildlife Service, Dubbo, NSW. http://www.nationalparks.nsw.gov.au/PDFs/ NSWNPWS_drought_harvestmanagement_colour.pdf

Pople, A. R. (2006). Modelling the spatial and temporal dynamics of kangaroo populations for harvest management. Final report to the Department of Environment and Heritage, Canberra.

Pople, A. R., and Grigg, G. C. (1998). Commercial harvesting of kangaroos in Australia. Environment Australia, Canberra. http://www.ea.gov.au/ biodiversity/trade-use/wild-harvest/kangaroo/harvesting/index.html

Robertson, G. G. (1986). The mortality of kangaroos in drought. Australian Wildlife Research 13, 349-354. doi:10.1071/WR9860349

Robertson, G. (1987). Plant dynamics. In 'Kangaroos: their Ecology and Management in the Sheep Rangelands of Australia'. (Eds G. Caughley, N. Shepherd and J. Short.) pp. 50-68. (Cambridge University Press: Cambridge.)

Williams, B. K., Conroy, M. J., and Nichols, J. D. (2002). 'Analysis and Management of Animal Populations: Modeling, Estimation, and Decision Making.' (Academic Press: San Diego, CA.)

Manuscript received 8 June 2007, accepted 24 December 2007 